\title{
Mixture Toxicity of Methylisothiazolinone and Propylene Glycol at a Maximum Concentration for Personal Care Products
}

\author{
Juyoung Park, Handule Lee and Kwangsik Park \\ College of Pharmacy, Dongduk Women's University, Seoul, Korea
}

\begin{abstract}
Methylisothiazolinone (MIT) has been used in combination with methylchloroisothiazolinone (CMIT) for cosmetic products such as shampoo, body lotion, and skin care products. The mixture of CMIT/MIT has been found to cause allergic contact dermatitis and is thus no longer permitted for use as a preservative in leave-on cosmetics. However, MIT itself was approved as a stand-alone preservative at a maximum concentration of $100 \mathrm{ppm}$ as the toxicity was derived from CMIT rather than MIT. However, in many countries, allergic skin irritation caused by MIT remains a social concern. In this study, skin irritation was assessed for the presence of MIT, propylene glycol, and their mixture using a 3D human skin model EpiDerm ${ }^{\mathrm{TM}}$. Although non-diluted MIT causes serious skin toxicity, skin irritation was not observed at a concentration of $100 \mathrm{ppm}$, the maximum permissible level for cosmetics and personal care products according to European regulations. Propylene glycol, the most widely used vehicle for MIT, did not cause skin irritation in the 3D skin model. The results are expected to provide information for regulatory policies and guidelines on the use of biocides in consumer products.
\end{abstract}

Key words: Methylisothiazolinone, Propylene glycol, Skin irritation, Mixture toxicity

\section{INTRODUCTION}

Methylchloroisothiazolinone (CMIT) and methylisothiazolinone (MIT) are heterocyclic organic compounds that have long been used in cosmetic products such as shampoo, body lotion, and skin care products $(1,2)$ under the name of Kathon ${ }^{\mathrm{TM}}$ CG. With increasing uses of CMIT/ MIT, toxicity studies have been widely performed to predict human health risks, particularly skin irritation and sensitization risks $(3,4)$. Adverse effects of Kathon ${ }^{\mathrm{TM}} \mathrm{CG}$ are known to be caused mainly by CMIT rather than by MIT $(5,6)$. Therefore, the use of CMIT in leave-on products is prohibited. Now, only MIT is permitted to be used in cosmetics and many other personal care products. Gen-

Correspondence to: Kwangsik Park, College of Pharmacy, Dongduk Women's University, 60, Hwarang-ro, 13-gil, Seongbuk-gu, Seoul 02748, Korea

E-mail: kspark@dongduk.ac.kr

This is an Open-Access article distributed under the terms of the Creative Commons Attribution Non-Commercial License (http:// creativecommons.org/licenses/by-nc/3.0) which permits unrestricted non-commercial use, distribution, and reproduction in any medium, provided the original work is properly cited. eral uses of MIT have widely increased, including household cleaning products and paints (7). A study from 2015 showed that $93.0 \%$ of the paints in five European countries contain MIT at concentrations ranging from 1.1 to 142.7 ppm (8).

However, recent epidemiological studies have shown that the use of MIT alone can cause contact allergic dermatitis $(9,10)$. A retrospective analysis of data collected by the European Surveillance System on the contact allergies of 31,689 consecutively patch-tested patients from 46 departments in 12 European countries revealed that the prevalence of contact allergy to methylisothiazolinone has increased by up to around $20 \%$ in several departments (11). In 9 dermatology clinics representing 9 countries, 3865 consecutive patients with dermatitis were patch tested with $0.2 \%$ MIT. The prevalence of the contact allergy to MIT in these participating clinics was found in 284 (7.3\%) patients (12). In animal tests, potential sensitization has been suggested when mice were treated with methylisothiazolinone (13). Dermal treatment with MIT in both the $\mathrm{C} 57 \mathrm{BL} / 6$ and $\mathrm{BALB} / \mathrm{c}$ mice has induced increases of Tand B-cell proliferation in the auricular lymph nodes along with IFN- $\gamma$ production and limited increases in total serum $\mathrm{IgE}$, thus confirming dermal sensitization. An airway chal- 
lenge with MIT has led to an early ventilatory response (decreased breathing frequency), indicative of acute sensory irritation (14). While the potent skin sensitization effect of MIT has been suggested, animal toxicity data on skin irritation to MIT alone are insufficient (15).

Recently, new issues on the toxicity of preservative mixtures that include MIT have been highlighted for biocide regulation in many countries. In terms of vehicles for active ingredient, propylene glycol (PG) is one of the most widely used solvents. PG is an aliphatic alcohol that functions as a skin conditioning agent, viscosity decreasing agent, solvent, and fragrance ingredient in cosmetics (16). PG is generally known to be non-toxic and non-carcinogenic. Clinical studies on PG have demonstrated an absence of dermal sensitization at the concentrations used, although concerns remain about its irritation. The safety of PG and MIT when mixed has not yet been reported.

In this study, the skin irritation capacities of MIT and PG alone and in combination (MIT/PG) were tested using a 3D human skin model. The results of this study are expected to provide information on the safety level of MIT and PG at the maximum concentration found in cosmetics and personal care products. They are also expected to provide information for regulatory policies and guidelines on the use of biocides in consumer products.

\section{MATERIALS AND METHODS}

Determining the optimal MIT and PG concentrations in a human keratinocyte cell line (HaCaT). HaCaT cells derived from human keratinocytes were cultured in Dulbecco's Modified Eagle Medium (DMEM) containing $10 \%$ heat-inactivated fetal bovine serum (FBS) and 1\% penicillin and streptomycin $(100 \mathrm{U} / \mathrm{mL}$ penicillin and 100 $\mu \mathrm{g} / \mathrm{mL}$ streptomycin) at $37^{\circ} \mathrm{C}$ with $5 \% \mathrm{CO}_{2}$. Cells were seeded into each well of a 96-well plate at a density of $6 \times$ $10^{5} \mathrm{cell} / \mathrm{mL}$ and incubated at $37^{\circ} \mathrm{C}$ for $24 \mathrm{hr}$. MIT (200, $300,400,500$, and $600 \mu \mathrm{M})$ and PG $(0.1,0.5,1,2$, and $4 \%$ ) were used to treat cultured cells. After $24 \mathrm{hr}$ of treatment, cell viability was measured by a colorimetric assay using 3-(4,5-dimethylthiazol-2-yl)-2,5-diphenyltetrazolium bromide (MTT).

Skin irritation test and reconstructed human epidermis (RhE). A skin irritation test was performed based on the Organisation for Economic Co-operation and Development (OECD) TG 439 or the manufacturer's protocol. Reconstructed human epidermis EpiDerm ${ }^{\mathrm{TM}}$ was purchased from MatTek Corporation (Ashland, MA, USA). EpiDerm ${ }^{\mathrm{TM}}$ is a ready-to-use highly differentiated $3 \mathrm{D}$ tissue model consisting of normal human-derived epidermal keratinocytes (NHEK) cultured on specially prepared tissue culture inserts. When cultured at an air-liquid interface (ALI), EpiDerm $^{\mathrm{TM}}$ enables the evaluation of topically applied compounds, chemicals, cosmetic/personal care product ingredients, and final formulations. EpiDerm ${ }^{\mathrm{TM}}$ exhibits a human epidermal tissue structure and cellular morphology with great uniformity and reproducibility. EpiDerm ${ }^{\mathrm{TM}}$ is a $3 \mathrm{D}$ structure consisting of organized and proliferative basal cells, spinous and granular layers, and cornified epidermal layers that are mitotically and metabolically active. After receiving the skin model product (EpiDerm ${ }^{\mathrm{TM}}$ ), tissue inserts were transferred to each well of a 6-well plate containing $0.9 \mathrm{~mL}$ DMEM and then pre-incubated at $37^{\circ} \mathrm{C}$ with $5 \% \mathrm{CO}_{2}$ overnight. The tissues were then distributed into three groups: (1) the negative control (NC) group, (2) $5 \%$ sodium dodecyl sulfate (SDS) as the positive control (PC) group, and (3) the test chemical-treated group. After the skin tissues were applied to the test chemicals, they were covered with nylon mesh and incubated for $1 \mathrm{hr}$ based on the manufacturer's test protocol. After the exposure time, tissues were washed with phosphate buffered saline (PBS) and post-incubated for $42 \mathrm{hr}$. The cell viability of the skin tissue was then measured with the MTT method as previously described (17-19).

Classification of skin irritation. MTT ( $1 \mathrm{mg} / \mathrm{mL})$ solution was added to the post-incubated skin tissues and maintained at $37^{\circ} \mathrm{C}$ with $5 \% \mathrm{CO}_{2}$ for $3 \mathrm{hr}$. The absorbance was then measured by a colorimetric assay. The cell viability of the skin tissue was determined as a percentage of the absorbance of the test chemical group compared to that of the negative control group. Classification of skin irritation was performed according to the viability based on OECD TG 439. When the viability of a test group was less than $50 \%$, the chemical was classified as an irritant, (United Nations Global Harmonization System [UN GHS] category 2 or 1 ). If the viability was greater than $50 \%$, it was classified as a non-irritant chemical (Table 1).

IL-1 a assay using culture media of EpiDerm ${ }^{T M}$. After $1 \mathrm{hr}$ of exposure of EpiDerm ${ }^{\mathrm{TM}}$ to test the chemicals, the cells were washed with PBS and then post-incubated at $37^{\circ} \mathrm{C}$ with $5 \% \mathrm{CO}_{2}$ for $42 \mathrm{hr}$. At $24 \mathrm{hr}$ post-incubation, a small amount of the media was extracted and the level of interleukin-1 alpha (IL-1 $\alpha$ ) was measured using the IL-1 $\alpha$

Table 1. Classification criteria of skin irritation test defined in OECD TG 439

\begin{tabular}{ll}
\hline \hline \multicolumn{1}{c}{ Classification } & \multicolumn{1}{c}{ Viability } \\
\hline Irritant (UN GHS; Category 2) & Below defined threshold levels $(\leq 50 \%)$ \\
Non-irritant (UN GHS; No Category) & Above the defined threshold levels $(>50 \%)$ \\
\hline
\end{tabular}


assay kit (R\&D Systems, Minneapolis, MN, USA) according to the manufacturer's protocol. Briefly, capture antibody diluted in Dulbecco's PBS was dispensed into a 96well plate and incubated overnight at room temperature to be coated. Each well was washed with a wash buffer. The diluted reagent was then added to each well and incubated at room temperature. After washing, the media taken from the EpiDerm ${ }^{\mathrm{TM}}$ incubation were added to each well and incubated at room temperature. After incubation, each well was washed with wash buffer and detection antibody was added to each well. Subsequently, each well was treated with streptavidin-horseradish peroxidase (HRP) secondary antibody, substrate solution, and stop solution. The absorbance of each well was then measured at a wavelength of $450 \mathrm{~nm}$ on a microplate reader.

Statistics. All statistical analyses were performed using SPSS version 23 (IBM, Armonk, NY, USA). An independent two sample $t$-test was used to compare the means of the control and treated groups. Statistical significance was considered at $p<0.05$.

\section{RESULTS}

Cytotoxicity of MIT and PG in cultured HaCaT cells. The skin is the primary target of MIT in cosmetics or personal health care products (rinse-off and leave-on). Therefore, HaCaT cells derived from human keratinocytes could be appropriate for determining the concentration range of MIT in which skin irritation might occur. As shown in Fig. 1, cell viability decreased by treatment with MIT for $24 \mathrm{hr}$ in a concentration-dependent manner. No cytotoxic-

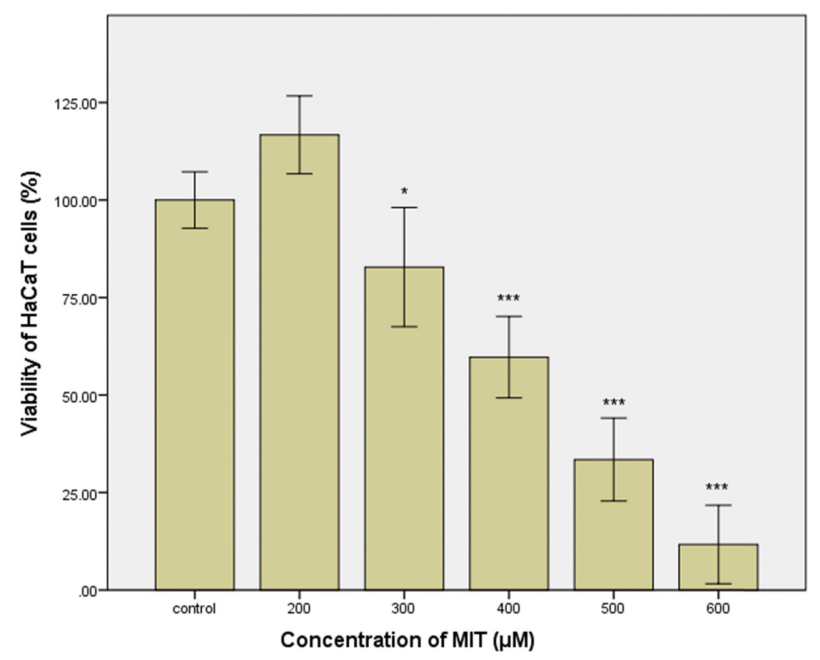

Fig. 1. Viability of cultured $\mathrm{HaCaT}$ cells treated with methylisothiazolinone (MIT) for $24 \mathrm{hr}$. Statistical analysis was performed by independent two sample $t$-test between the control group and the respective MIT-treated group. ${ }^{*} p<0.05 ;{ }^{* * *} p<0.001$.

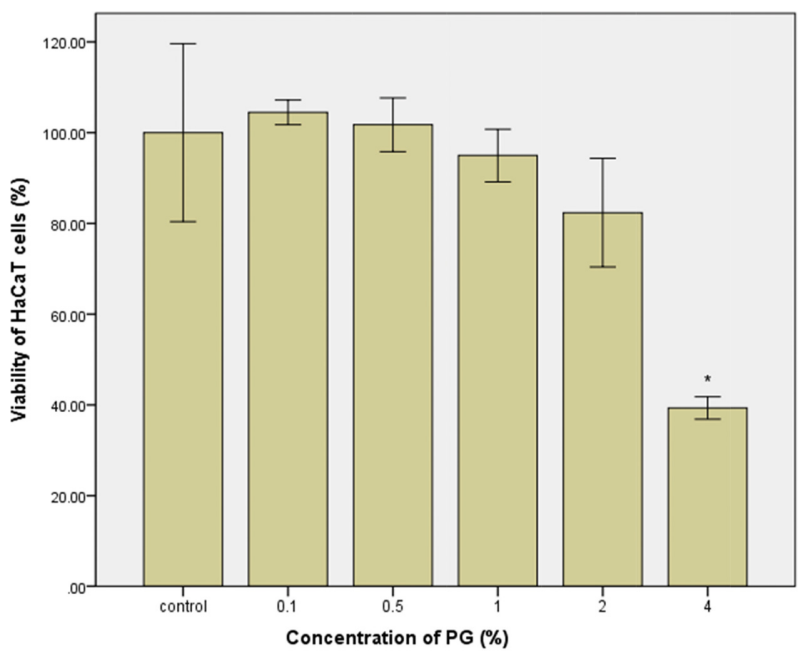

Fig. 2. Viability of cultured HaCaT cells treated with propylene glycol (PG) for $24 \mathrm{hr}$. Statistical analysis was performed by the independent two sample $t$-test for the control group and respective PG treated group. ${ }^{*} p<0.05$.

ity was observed for MIT at $200 \mu \mathrm{M}$. However, cell viability decreased gradually to $82.8 \%$ in cells treated with MIT at $300 \mu \mathrm{M}$, and decreased to $11.7 \%$ in cells treated with MIT at $600 \mu \mathrm{M}$. A small increase in the MIT concentration can result in a significant increase of cytotoxicity. PG, the vehicle of MIT, seems to show the same concentration-dependent cytotoxicity after treatment for $24 \mathrm{hr}$. No cytotoxicity was observed in the treatment with PG at a concentration of $0.5 \sim 2 \%$. However, the cell viability decreased to $39.3 \%$ after treatment with PG at $4 \%$ (Fig. 2).

Skin irritation of MIT. A cytotoxicity test using cultured $\mathrm{HaCaT}$ cells showed a cell viability of $11.7 \%$ in the group treated with MIT at $600 \mu \mathrm{M}(69.1 \mathrm{ppm})$ for $24 \mathrm{hr}$. Therefore, two test concentrations of $0.01 \%$ (100 ppm) and $1 \%(10,000 \mathrm{ppm})$ were selected for a skin irritation test of MIT in EpiDerm ${ }^{\mathrm{TM}}$. The exposure time of MIT in the EpiDerm ${ }^{\mathrm{TM}}$ test was $1 \mathrm{hr}$, which was very short compared to that in the cell viability test using HaCaT. MIT was solubilized in DMEM and applied to EpiDerm ${ }^{\mathrm{TM}}$ for $1 \mathrm{hr}$. As shown in Fig. 3, MIT itself (100\% solution) showed strong toxicity to EpiDerm ${ }^{\mathrm{TM}}$, indicating that MIT was a strong irritant. However, MIT did not show cytotoxicity at a concentration of $0.01 \%(100 \mathrm{ppm})$, which is the maximum permissible concentration in personal health care products. This suggests that MIT in this concentration does not cause skin irritation. After MIT was solubilized in $\mathrm{PG}$ and applied to EpiDerm ${ }^{\mathrm{TM}}$ for $1 \mathrm{hr}$, $\mathrm{PG}$ alone showed no toxic effects in the skin models (Fig. 4). The cytotoxicity of MIT dissolved in PG (Fig. 4) was almost the same as that of MIT dissolved in DMEM without PG (Fig. 3). MIT did not show cytotoxicity at a concentration of $0.01 \%$ 


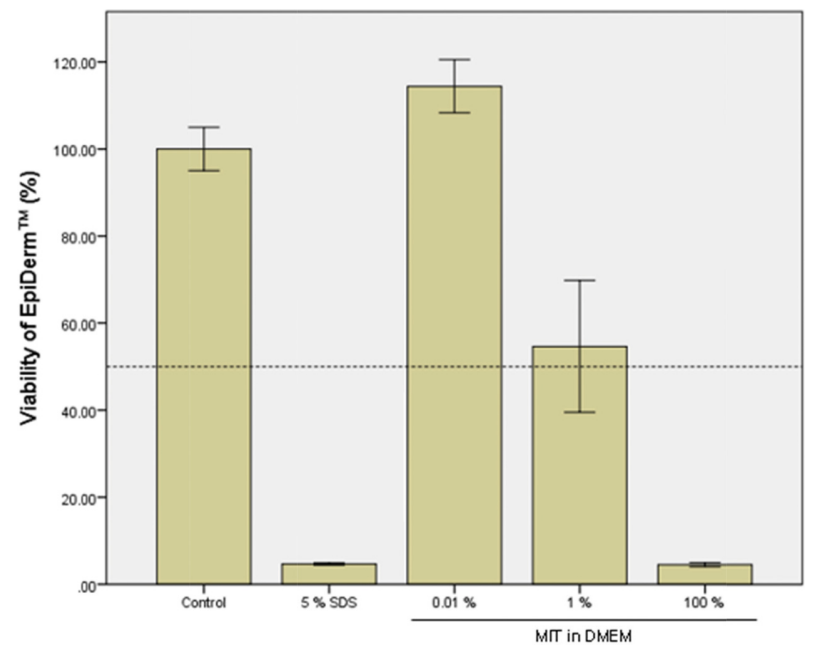

Fig. 3. Skin irritation test of methylisothiazolinone (MIT) solubilized in Dulbecco's Modified Eagle Medium (DMEM) using the EpiDerm ${ }^{T M}$ 3D human skin model. EpiDerm ${ }^{T M}$ 3D human skin models were treated with MIT solubilized in DMEM for $1 \mathrm{hr}$, and the cell viability of the skin model was measured by MTT assay. The control group was treated with only PBS without MIT, and 5\% SDS was used as the positive chemical.

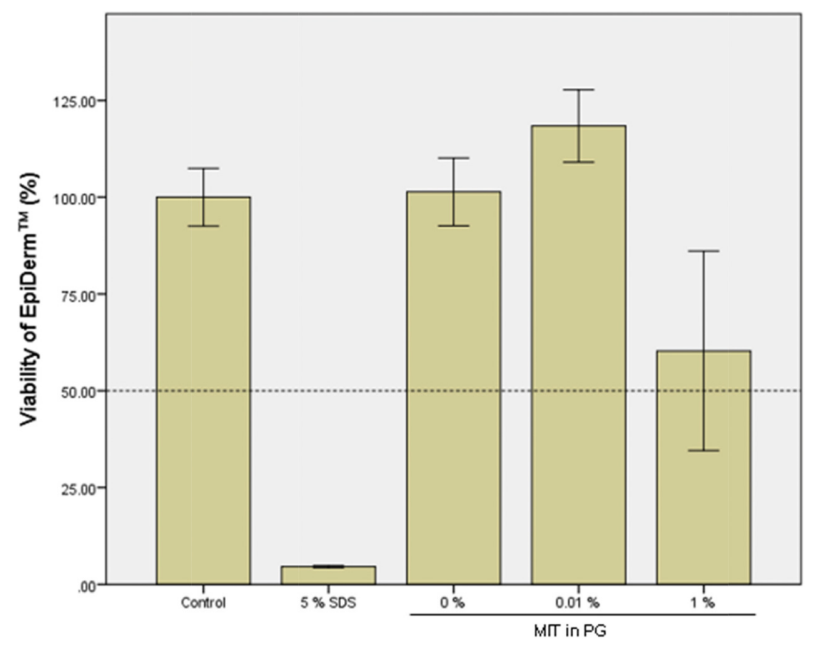

Fig. 4. Skin irritation test of methylisothiazolinone (MIT) solubilized in propylene glycol (PG) using EpiDerm ${ }^{\mathrm{TM}} 3 \mathrm{D}$ human skin model. EpiDerm ${ }^{\mathrm{TM}} 3 \mathrm{D}$ human skin models were treated with MIT solubilized in PG for $1 \mathrm{hr}$, and the cell viability was measured by the MTT assay. The control group was treated with phosphate buffered saline without MIT, and 5\% SDS was used as a positive chemical.

(100 ppm) when it was solubilized in PG. This means that PG does not have any additive effect on the cytotoxicity of EpiDerm $^{\mathrm{TM}}$ caused by MIT.

Toxicity of the MIT and PG mixture. The toxicity of the MIT and PG mixture to skin irritation was not observed

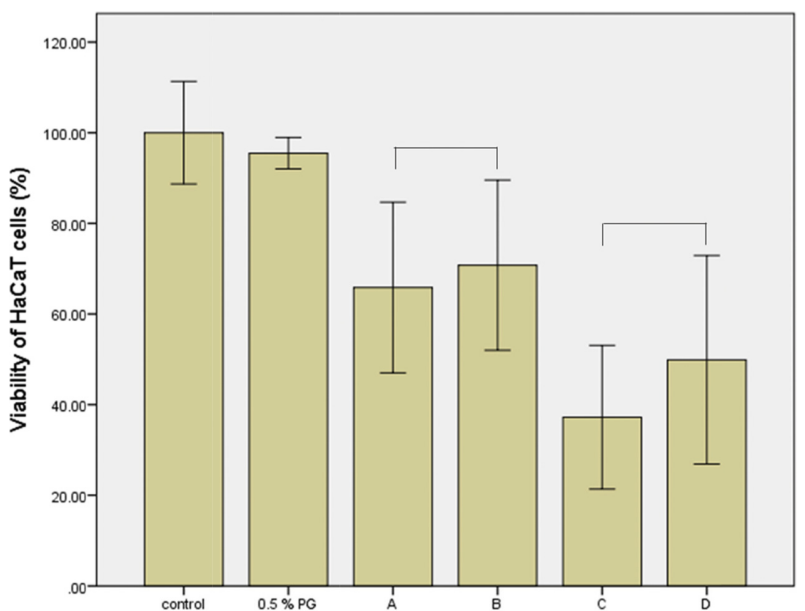

Fig. 5. Mixture toxicity of methylisothiazolinone (MIT) and $0.5 \%$ propylene glycol (PG) to cultured $\mathrm{HaCaT}$ cells. Cultured $\mathrm{HaCaT}$ cells were treated with a mixture of MIT and PG for $24 \mathrm{hr}$, and viability was measured by MTT assay. Cell viabilities did not decrease in the $0.5 \%$ PG-treated or negative control groups. MIT (A, $300 \mu \mathrm{M} ; \mathrm{C}, 400 \mu \mathrm{M})$ showed cytotoxicity in a concentration-dependent manner. Its toxicity was not influenced by 0.5 $\%$ PG. A, $300 \mu \mathrm{M}$ MIT; B, $300 \mu \mathrm{M}$ MIT + 0.5\% PG; C, $400 \mu \mathrm{M}$ MIT; $\mathrm{D}, 400 \mu \mathrm{M} \mathrm{MIT}+0.5 \%$ PG. There was no statistically significant $(p>0.05)$ difference between $A$ and $B$, or between $C$ and $D$.

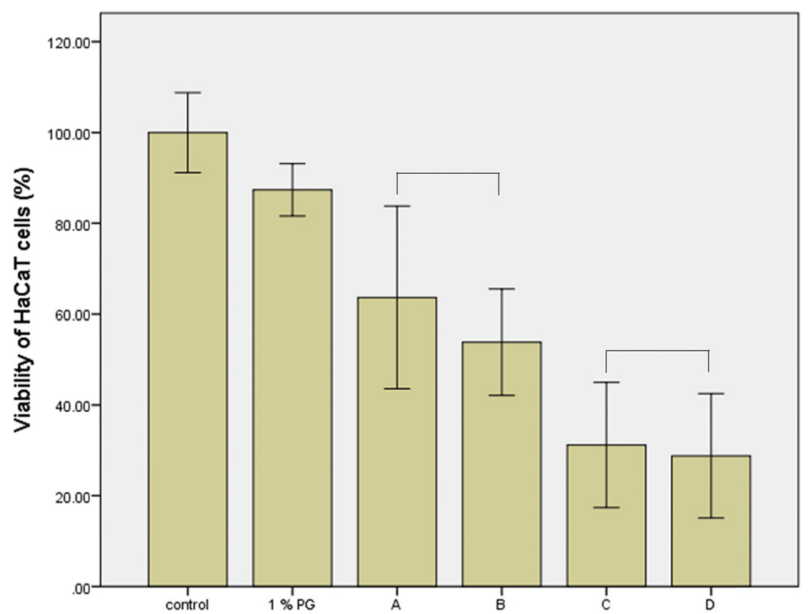

Fig. 6. Mixture toxicity of methylisothiazolinone (MIT) and 1\% propylene glycol (PG) to cultured $\mathrm{HaCaT}$ cells. Cultured $\mathrm{HaCaT}$ cells were treated with a mixture of MIT and PG for $24 \mathrm{hr}$, and viability was measured by the MTT assay. Cell viabilities did not decrease in the $1 \%$ PG-treated or negative control groups. MIT (A, $300 \mu \mathrm{M} ; \mathrm{C}, 400 \mu \mathrm{M})$ showed cytotoxicity in a concentrationdependent manner. Its toxicity was not influenced by the $1 \%$ PG. A, $300 \mu \mathrm{M}$ MIT; B, $300 \mu \mathrm{M}$ MIT + 1\% PG; C, $400 \mu \mathrm{M}$ MIT; D, $400 \mu \mathrm{M}$ MIT $+1 \%$ PG. No statistically significant $(p>0.05)$ difference was observed between $A$ and $B$, or between $C$ and $D$.

using the 3D human skin model, as shown in Fig. 3 and Fig. 4. The mixture also had no effect on the viability of 


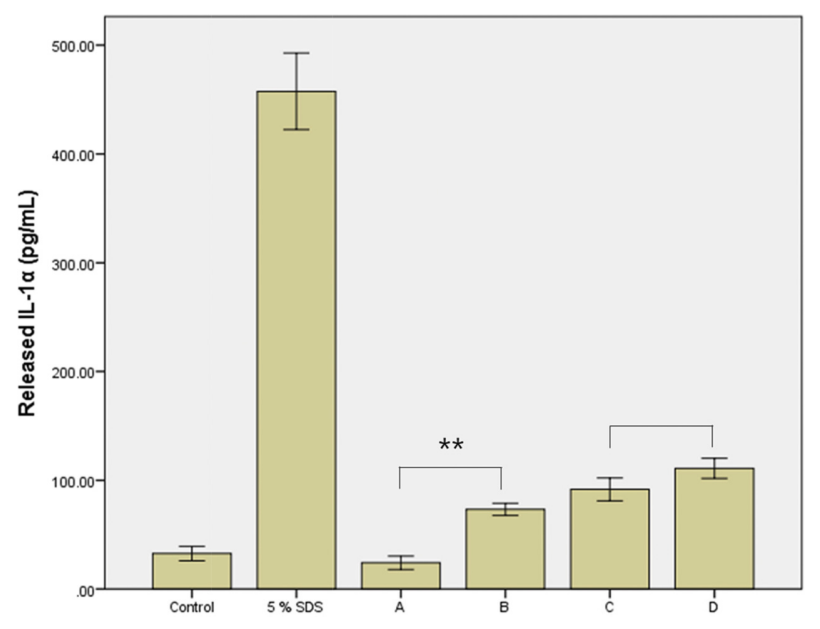

Fig. 7. Released IL-1 $\alpha$ in the culture medium of EpiDerm ${ }^{\mathrm{TM}}$ treated with methylisothiazolinone (MIT) alone or its mixture with propylene glycol (PG). At $24 \mathrm{hr}$ post-treatment, a small amount of the media was extracted and the IL-1 $\alpha$ level was measured. In the positive control group treated with 5\% SDS, a high level of IL-1 $\alpha$ was detected. IL- $1 \alpha$ was not induced by $0.01 \%$ MIT. However, it was increased by the mixture of PG and MIT $(p<0.01)$. A, 0.01\% MIT in DMEM; B, 0.01\% MIT in PG; C, $1 \%$ MIT in DMEM; D, $1 \%$ MIT in PG. ${ }^{* *} p<0.01$.

cultured HaCaT cells (Fig. 5, 6). The cytotoxicity of MIT (300 or $400 \mu \mathrm{M}$ ) according to vehicle concentration did not change. PG at a concentration of $0.5 \%$ or $1 \%$ did not affect the cytotoxicity of MIT. However, the inflammatory response assay for IL-1 $\alpha$, a cell mediator related to skin irritation and released from the skin model, differed to that of the previous results. As shown in Fig. 7, the level of IL-1 $\alpha$ did not increase in the skin tissue treated with $0.01 \%$ MIT (100 ppm) when MIT was solubilized in DMEM without the PG vehicle. However, it increased when MIT was solubilized in the PG vehicle. However, $1 \%$ MIT (10,000 ppm) solubilized in DMEM without the PG vehicle increased the level of IL- $1 \alpha$, while the synergistic effect of PG was not as high as that in the $0.01 \%$ MIT-treated skin model.

\section{DISCUSSION}

Market surveys have shown that a mixture of CMIT, MIT, oligo(2-)ethoxy ethoxyethyl guanidine chloride (PGH), benzalkonium chloride, and PHMG has been used as a humidifier disinfectant in Korea (20,21), although all such products are no longer commercially available. However, several of these products still have general uses in personal care products. For example, in many countries, CMIT/ MIT has long been used in cosmetic products, such as shampoo, body lotion, and skin care, as well as in paint manufacturing and as biocides in cooling towers $(1,2)$. Therefore, toxicity studies have been widely performed to predict the effects of these substances on the human body, particularly focusing on skin irritation. Patch test files and clinical records of patients examined at the Finnish Institute of Occupational Health between January 2002 and February 2013 demonstrated that 72 (4.1\%) of 1,745 patients showed allergic reaction to CMIT/MIT and/or MIT. Positive patch test reactions to CMIT/MIT were observed in $45(2.6 \%)$ patients. Positive reactions to both CMIT/MIT and MIT were shown in $24(1.4 \%)$ patients, while positive reactions to MIT were only observed in $3(0.2 \%)$ patients (22). Another patch test for CMIT/MIT was performed on 1,498 Swedish patients with suspected allergic contact dermatitis. It was found that $7.1 \%$ of these patients responded to CMIT/MIT (23).

In the present study, it was found that non-diluted MIT was very toxic to the skin model. Even when it was diluted to $50 \%$ with DMEM, it remained toxic to the skin model. All of the cells in the tissue appeared to have died (data not shown). According to the classification based on OECD TG 439, MIT itself is a strong skin irritant. However, there has been no evidence demonstrating that MIT could cause skin irritation at a concentration of $0.01 \%(100 \mathrm{ppm})$, the maximum permissible concentration by European regulations (Fig. 3). When the test concentration of MIT was increased to $1 \%$, skin irritancy seemed to be uncertain. Further data are needed because the viability was borderline for classifications. The standard deviation of MIT was relatively large compared to that of other groups. All viability results were obtained from three skin tissues, except for the $1 \%$ MIT group, for which results were obtained from six skin tissues. Although $100 \mathrm{ppm}$ of MIT did not result in irritation, this does not imply that this MIT concentration is safe. In a skin irritation test using 3D human skin models, the skin was exposed to the test chemical for only $1 \mathrm{hr}$. Therefore, results from chronic exposure cannot be predicted. This aspect has also been considered in tests in other studies $(24,25)$. While non-diluted MIT showed strong cytotoxicity in skin models, non-diluted PG itself did not show cytotoxicity in the skin model (Fig. $3,4)$ yet showed cytotoxicity to $\mathrm{HaCaT}$ cells (Fig. 2). When HaCaT cells were treated with PG at $4 \%$ for $24 \mathrm{hr}$, the cell viability was less than $40 \%$. However, there were no cytotoxic effects in the skin tissue model even after the skin tissue was treated with $100 \%$ PG for $1 \mathrm{hr}$. As shown in Fig. 3 to Fig. 6, the toxicities of the MIT and PG mixture were less apparent in the skin model as well as in $\mathrm{HaCaT}$ cells.

The effect of PG on the level of released cytokines seemed to differ from the effect of PG on cell viability. IL$1 \alpha$ was produced not only by activated macrophages, but also by neutrophils, epithelial cells, and endothelial cells. It has been known to play a role in inflammation initiation, fever, and progression of sepsis. In the SDS-treated positive control, the level of released IL- $1 \alpha$ was signifi- 
cantly increased. After treatment with MIT mixed with DMEM, the IL- $1 \alpha$ level did not increase in the $0.01 \%$ MIT-treated group (100 ppm). However, it was slightly increased in the $1 \%$ MIT-treated group $(10,000 \mathrm{ppm})$. When MIT was mixed with PG, the IL- $1 \alpha$ level was increased in both the $0.01 \%$ MIT-treated group (100 ppm) and the $1 \%$ MIT-treated group (10,000 ppm) (Fig. 7). This suggests a potential mixture toxicity of MIT with PG in inflammatory responses. However, the potentiation of the inflammatory response appears to be insufficient to induce skin irritation in EpiDerm ${ }^{\mathrm{TM}}$.

In conclusion, $100 \%$ MIT showed skin irritation in the 3D human skin model. PG itself did not cause skin irritation, nor did it show an additive or synergic effect on MITinduced skin irritation. However, it might have potential to induce inflammatory response when mixed with MIT. This finding needs further assessment using an in vivo system.

\section{ACKNOWLEDGMENTS}

This research was supported by the National Institute of Environmental Research and by the Korea Ministry of Environment (MOE) through the Environmental Health Action Program (2017001360001).

Received February 28, 2018; Revised May 15, 2018; Accepted July 4, 2018

\section{REFERENCES}

1. de Groot, A.C. and Weyland, J.W. (1988) Kathon CG: a review. J. Am. Acad. Dermatol., 18, 350-358.

2. Yazar, K., Johnsson, S., Lind, M.L., Boman, A. and Lidén, C. (2011) Preservatives and fragrances in selected consumeravailable cosmetics and detergents. Contact Derm., 64, 265 272.

3. Concha-Garzón, M.J., Solano-López, G., Montes, A., Fraga, J. and Sánchez, J. (2015) Follicular allergic contact dermatitis due to methylchloroisothiazolinone/methylisothiazolinone (MCI/MI) in a rinse-off soap product. Clin. Exp. Dermatol., 40, 690-691.

4. Liuti, F., Hernández, H.Z. and Borrego, H.L. (2014) Increased sensitization to Kathon CG (methylchloroisothiazolinone plus methylisothiazolinone) in the south of Gran Canaria, Spain. Actas Dermosifiliogr., 105, 882-883.

5. Hou, F., Xing, C., Li, B., Cheng, J., Chen, W. and Zhang, M. (2015) Application of BALB/c mouse in the local lymph node assay: BrdU-ELISA for the prediction of the skin sensitizing potential of chemicals. J. Pharmacol. Toxicol. Methods, 72, 53-58.

6. Gerberick, G.F., Ryan, C.A., Kimber, I., Dearman, R.J., Lea, L.J. and Basketter, D.A. (2000) Local lymph node assay: validation assessment for regulatory purposes. Am. J. Contact Dermat., 11, 3-18.

7. Mary, A.L. (1992) Final report on the safety assessment of methylisothiazolinone and methylchloroisothiazolinone. $J$.
Am. Coll. Toxicol., 11, 75-128.

8. Thomsen, A.V., Schwensen, J.F., Bossi, R., Banerjee, P., Giménez-Arnau, E., Lepoittevin, J.P., Lidén, C., Uter, W., White, I.R. and Johansen, J.D. (2017) Isothiazolinones are still widely used in paints purchased in five European countries: a follow-up study. Contact Derm., 78, 246-253.

9. Aerts, O., Goossenc, A. and Giordano-Labadie, F. (2015) Contact allergy caused by methylisothiazolinone: the Belgian-French experience. Eur. J. Dermatol., 25, 228-233.

10. Ali, F.R., Shephered, E.L., Yell, L.C., Buckley, D.A. and Williams, J.D. (2014) Escalating methylisothiazolinone/ methylchloroisothiazolinone allergy probably attributable to methylisothiazolinone in leave-on body cosmetics. Contact Derm., 70, 316-317.

11. Uter, W., Amario-Hita, J.C., Balato, A., Ballmer-Weber, B., Bauer, A., Belloni, F.A., Bircher, C.M.M.U., Cooper, S.M., Czarnecka-Operacz, M., Dugonik, A., Gallo, R., GiménezArnau, A., Johansen, J.D., John, S.M., Kieć-Świerczyńska, M., Kmecl, T., Kręcisz, B., Larese, F.F., Mahler, V., Pesonen, M., Rustemeyer, T., Sadowska-Przytocka, A., Sánchez-Pérez, J., Schliemann, S., Schuttelaar, M.L., Simon, D., Spiewak, R., Valiukevičiene, S., Weisshaar, E., White, I.R. and Wilkinson, S.M. (2017) European Surveillance System on Contact Allergies (ESSCA): results with the European baseline series, 2013/14. J. Eur. Acad. Dermatol. Venereol., 31, 15161525.

12. Isaksson, M., Ale, I., Andersen, K.E., Elsner, P., Goh, C.L., Goossens, A., Jerajani, H., Matsunaga, K., McFadden, J. and Bruze, M. (2017) Multicenter patch testing with methylisothiazolinone and methylchloroisothiazolinone/methylisothiazolinone within the international contact dermatitis research group. Dermatitis, 28, 210-214.

13. Warbrick, E.V., Dearman, R.J., Basketter, D.A. and Kimber, I. (1999) Influence of application vehicle on skin sensitization to methylchloroisothiazolinone/methylisothiazolinone: an analysis using the local lymph node assay. Contact Derm., 41, 325-329.

14. Devos, F.C., Pollaris, L., Van Den Broucke, S., Seys, S., Goossens, A., Nemery, B., Hoet, P.H. and Vanoirbeek, J.A. (2015) Methylisothiazolinone: dermal and respiratory immune responses in mice. Toxicol. Lett., 235, 179-188.

15. Miles, A., Berthet, A., Hopf, N.B., Gilliet, M., Raffoul, W., Vernez, D. and Spring, P. (2014) A new alternative method for testing skin irritation using a human skin model: a pilot study. Toxicol. In Vitro, 28, 240-247.

16. McGowan, M.A., Scheman, A. and Jacob, S.E. (2017) Propylene glycol in contact dermatitis: a systematic review. Dermatitis, 29, 6-12.

17. OECD (2015) OECD Guidelines for the Testing of Chemicals, Section 4. Test No. 439, In Vitro Skin Irritation: Reconstructed Human Epidermis Test Method. doi: 10.1787/ 9789264242845-en/.

18. Kim, H., Choi, J., Lee, H., Park, J., Yoon, B.I., Jin, S.M. and Park, K. (2016) Skin corrosion and irritation test of nanoparticles using reconstructed three-dimensional human skin model, EpiDerm ${ }^{\mathrm{TM}}$. Toxicol. Res., 32, 311-316.

19. Choi, J., Kim, H., Choi, J., Oh, S.M., Park, J. and Park, K. (2014) Skin corrosion and irritation test of sunscreen nanoparticles using reconstructed 3D human skin model. Environ. 
Health Toxicol., 29, e2014004.

20. Park, D., Ryu, S., Lim, H., Kim, S., Roh, H., Cha, W. and Park, D.Y. (2016) Estimation of humidifier disinfectant amounts inhaled into the respiratory system. J. Environ. Health Sci., 42, 141-146.

21. Yoon, J., Cho, H.J., Lee, E., Choi, Y.J., Kim, Y.H., Lee, J.L., Lee, Y.J. and Hong, S.J. (2017) Rate of humidifier and humidifier disinfectant usage in Korean children: A nationwide epidemiologic study. Environ. Res., 155, 60-63.

22. Vauhkala, A.R., Pesonen, M., Suomela, S., Kuuliala, O., Suuronen, K. and Aalto-Korte, K. (2015) Occupational contact allergy to methylchloroisothiazolinone/methylisothiazolinone and methylisothiazolinone. Contact Derm., 73, 150-156.

23. Engfeldt, M., Bråred-Christensson, J., Isaksson, M., Matura,
M., Ryberg, K., Stenberg, B., Svedman, C. and Bruze, M. (2015) Swedish experiences from patch testing methylisothiazolinone separately. Acta Derm. Venereol., 95, 717-719.

24. Lee, M., Hwang, J.H. and Lim, K.M. (2017) Alternatives to in vivo draize rabbit eye and skin irritation tests with a focus on $3 \mathrm{~d}$ reconstructed human cornea-like epithelium and epidermis models. Toxicol. Res., 33, 191-203.

25. Macfarlane, M., Jones, P., Goebel, C., Dufour, E., Rowland, J., Araki, D., Costabel-Farkas, M., Hewitt, N.J., Hibatallah, J., Kirst, A., McNamee, P., Schellauf, F. and Scheel, J. (2009) A tiered approach to the use of alternatives to animal testing for the safety assessment of cosmetics: skin irritation. Regul. Toxicol. Pharmacol., 54, 188-196. 